\title{
Special issue on smart cameras for real-time image and video processing
}

\author{
Caifeng Shan ${ }^{1} \cdot$ Victor Manuel Brea ${ }^{2} \cdot$ Senem Velipasalar ${ }^{3}$
}

Published online: 4 August 2020

(c) Springer-Verlag GmbH Germany, part of Springer Nature 2020

Smart cameras and smart camera networks are becoming fundamental pieces for intelligent environments, ranging from homes to buildings and cities, progressively being inserted into our lives. From smart surveillance systems composed of a multitude of smart camera nodes to small wearable cameras able to render a visual log of our daily activities, these cameras interact with each other and with a wealth of other smart devices, and of course the Internet. Thanks to the convergence of relevant technologies such as imaging sensors, embedded systems, video analytics and deep learning, this field is currently undergoing a rapid development. This technology opens the door to many application domains including video surveillance, autonomous driving, robots and drones, smart factory and industry 4.0, health monitoring and care, to name a few. Real-time image and video processing play a key role in most of these applications.

The goal of this special issue is to present and highlight the latest developments in the smart camera community with the focus being on the real-time aspects of image and video processing. It attracted a large number of submissions from researchers active in the area of image processing and computer vision. After a careful peer review process, nine manuscripts were accepted for publication in this special issue, covering the topics of automatic camera calibration, image super-resolution, motion deblurring, multiple object tracking, face attribute recognition, human detection, image

Caifeng Shan

caifeng.shan@gmail.com

Victor Manuel Brea

victor.brea@usc.es

Senem Velipasalar

svelipas@syr.edu

1 Shandong University of Science and Technology, Qingdao, China

2 University of Santiago de Compostela, A Coruña, Spain

3 Syracuse University, Syracuse, NY, USA retrieval, and energy-efficient design of smart cameras. An overview of the nine accepted papers is provided below.

Camera calibration is a necessary preliminary step for computer vision in the 3D world. In particular, extrinsic camera parameters need to be computed each time a camera changes its position, thus not allowing for fast and dynamic network re-configuration. Many works have tried to automate the process of camera calibration. However, there is still a lack of fully unsupervised and markerless approaches for camera calibration in literature. Garau et al. present an unsupervised framework for estimating the extrinsic parameters of a camera network, which leverages an optimized 3D human mesh recovery from a single image, and does not require the use of additional markers. Their framework can work with a single camera in real-time, allowing the user to add, re-position, or remove cameras from the network in a dynamic fashion.

Ultra-High-Definition (UHD) cameras are commercially available, but still expensive for many applications. Superresolution (SR) techniques have been exploited to reconstruct high-resolution images or video without modifying the sensor architecture. In order to fix the artifacts observed in highly textured areas of images, Marin et al. propose a two-step SR method, called local adaptive spatial super resolution (LASSR). This method includes a machine learningbased texture analysis and a fast interpolation method that performs a pixel-by-pixel SR. They present an FPGA-based implementation of the LASSR method, which enables highquality 2-4 k super-resolution videos to be performed at 16 frames per second (fps), using only $13 \%$ of the FPGA capacity; this opens the way to reach more than 60 fps by executing several parallel instances of the LASSR code on the FPGA.

Recently, deep convolutional neural networks (CNNs) have been employed to handle low-level vision problems, such as resolution reduction and motion blurring. However, most existing CNN-based approaches can either handle single degeneration each time or treat them jointly through 
feature entangling, thus likely leading to poor performance when the actual degradation is inconsistent with the hypothetical degradation condition. Furthermore, feature coupling will result in a large amount of computation, which may make the methods impractical for real-time mobile scenarios. To address these problems, Liu et al. propose a deep decoupled cooperative learning model, which can not only develop the corresponding recovery network to deal with each degradation factor, but also flexibly handle multiple degradations at the same time. Their approach is exploited for simultaneous image super-resolution and motion deblurring.

As an important task in computer vision, multiple object tracking (MOT) has attracted much attention in recent years. Gong and Le present their implementation of multiple object tracking on vision digital signal processing (vision DSP). They investigate how to improve the speed performance and reduce system resource consumption under limited system resources. For data processing, they utilize the parallel characteristics of iDMA (integrated direct memory access) and a DSP core; as for data storage, a time-sharing strategy is proposed to solve the DSP local memory (data RAM) usage issue for tracking multiple objects. They also present a strategy for software design which includes two levels of parallel computations (frame-level and object-level).

Intelligent sports video analysis systems have many commercial applications, and spawned much research in recent years. With the development of accurate object detection and tracking algorithms in computer vision, one focus is on a detailed analysis of sports videos, such as player detection, identification and tracking. This can be used, for example, from the coaching staff's point of view, to gather game statistics and analyze the strengths and weaknesses of teams. $F u$ et al. present their study on tracking multiple players in the basketball game, as a practical application of MOT. They collect a data set of basketball game videos recorded in real life, and annotate the dataset for multiple-player tracking. They evaluate and compare several state-of-the-art MOT methods on the data set.

Recognizing face attributes (including gender, race, age, skin color, etc.) in the wild is a challenging problem. Compared to face recognition, face attribute-based methods are more relevant for many tasks. For instance, people of interest (criminal suspects or lost people) are always described by their identifiable facial attributes, such as big nose, high cheekbones, bushy eyebrows and so on. Yang et al. propose a horizontal pyramid global convolution (HPGC) module as a feature mapping operator to extract more local information for face attribute recognition. They design a lightweight attribute convolutional neural network (LACNN) combined with HPGC, using sigmoid cross entropy loss function. By replacing full connection or global average pooling with the proposed HPGC module, their approach balances the accuracy and computational efficiency.

The introduction of various multicore and GPU architectures has greatly enabled real-time implementation of video surveillance algorithms. Bahri et al. present a CUDA implementation of moving human detection on GPU, based on a robust approach capable of integrating global and local information by drawing upon the strengths of several visual features. More specifically, in their implementation, the moving object is first extracted by background subtraction based on the Gaussian Mixture Model, and then two complementary features (contour-based and region-based) are extracted for moving object classification using Support Vector Machine. The implementation on a GPU is 19 times faster than that on a CPU, and can detect pedestrians in real-time effectively.

Efficient image retrieval plays a key role in many applications. Hashing, which represents data with compact binary code, has attracted much attention in conducting efficient retrieval in the large-scale datasets via bit-wise calculations on the Hamming distance. $Q i$ et al. propose a deep hashing framework for medical image retrieval, referred to as supervised deep semantics-preserving hashing, where the process of deep feature extraction, binary code learning, and deep hash function learning are jointly carried out in a supervised fashion. Experiments on public dataset show that their method outperforms the-state-of-the-art methods.

With the rapid development of the Internet of Things, many changes have been introduced through the years in terms of design and architecture of vision sensor networks (VSN). Particularly for wireless VSN, it is necessary to investigate energy-efficient designs of smart cameras, enabling their implementation as battery-operated devices with a satisfactory lifetime. Based on a people counting scenario, Shallari et al. investigate how the allocation of image processing tasks on embedded hardware, software or cloud affects the overall node energy consumption. Their work not only provides an energy-efficient design for a people counting scenario, but also explores the trade-off between computation and communication energy consumption in a smart camera node for data-intensive IoT scenarios.

Acknowledgement The guest editors have many people to thank for their contributions to this special issue. We would like to thank all the authors for their innovative contributions, and all the reviewers for their professional, crucial, and constructive comments. In addition, we would like to thank the editorial team of the Journal of Real-Time Image Processing. We hope that you will enjoy this collection of articles, and that this special issue can spark and stimulate further research and development in this area.

Publisher's Note Springer Nature remains neutral with regard to jurisdictional claims in published maps and institutional affiliations. 